\title{
Achieving the Sustainable Development Goal 3: challenges in HIV testing in the Eastern Mediterranean Region
}

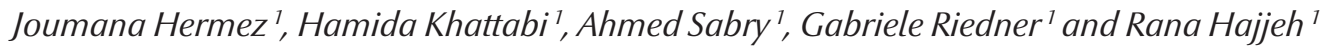

In the era prior to antiretroviral therapy (ART), taking an HIV test was associated with great fear, as a diagnosis of HIV infection signaled imminent death (1). As we mark World AIDS Day on 1 December 2017, it is worth reflecting on the success over the last two decades whereby treatment for HIV infection has become safer, more effective and simpler as new regimens with fixed combinations of antiretroviral drugs have become available (ARV). The use of ARVs has equally revolutionized HIV prevention beyond condom use, syringe exchange and screening of transfusion blood and transplant organs. ART suppresses viral replication, reduces viral load and thus minimizes the possibility of HIV transmission (1). Furthermore, pre and post-exposure prophylaxis with ARVs have proven efficacy in preventing infection of individuals exposed to HIV. Similarly, the advent of highly sensitive and specific rapid diagnostic tests and self-tests has improved access to quality testing services, and made it possible to return test results on the spot. Those scientific advances have put HIV testing and early diagnosis of HIV infection in a central position within the continuum of prevention, care and treatment.

The global response to HIV has translated those advances into largescale implementation of prevention, diagnosis, care and treatment services. As a result, the Joint United Nations Programme on HIV/AIDS (UNAIDS) announced in 2015 that the world has achieved and exceeded Millennium Goal 6 (MDG6) (2). This has given hope to the world to "end AIDS" by 2030 - one of the Sustainable Development Goals (SDG3).

However, in the Eastern Mediterranean Region (EMR) the HIV epidemic continues to grow. By the end of 2016, an estimated 360000 people were living with HIV in the Region, with 37000 new infections and 17000 deaths occurring in the same year (3). Over 95\% of new infections occurred among men who have sex with men, people who inject drugs, prisoners and sex workers (4). By the end of 2016, ART coverage in the EMR stood at $15 \%$. The biggest bottleneck to accessing treatment is the low coverage of HIV testing services. Between 2013 and 2015 several countries in the Region conducted HIV test-treat-retain cascade assessments, which have confirmed the challenges that countries face in identifying people living with HIV (PLHIV) (5). Between $50 \%$ and $93 \%$ of PLHIV remain undiagnosed and between 20\% and 50\% of persons diagnosed with HIV are lost-to-follow-up after diagnosis before enrolment in ART (5).

The reasons for low access to HIV testing include, but are not limited to, inadequate service delivery approaches, insufficient service availability, distance to testing services, cumbersome processes to get a final test result, poor referral services, weak community support, as well as long distances between testing and treatment facilities. Those challenges are exacerbated by stigma and discrimination against PLHIV and key populations - including in health care settings - punitive laws and criminalization of key populations. Of special concern is the late diagnosis of HIV, which results in death before or shortly after enrollment in care. Among the countries reporting their CD4 testing data, between $25 \%$ and $60 \%$ of PLHIV have CD 4 counts less than 200 cells / $\mu \mathrm{l}$ upon diagnosis, indicating late disease stage (6).

In 2016, countries of the EMR reported having conducted over 6.2 million HIV tests, excluding screening for blood donation. Of those, however, around two thirds were conducted in a mandatory manner on low-risk population groups (6). Only 3\% of the tests were conducted among key populations, $14 \%$ in health care settings and $13 \%$ in voluntary counselling and testing facilities (6). A negligible number of tests were conducted among partners of PLHIV. In contrast, $81 \%$ of PLHIV identified were from tests conducted among key populations, in health care settings and in voluntary counselling and testing facilities. This clearly demonstrates a skewed investment of resources towards less efficient testing approaches, focusing on less affected population groups. As a result, only 30\% of PLHIV know their HIV status, $54 \%$ of them (15\% of the total PLHIV) are receiving treatment and an unknown number is virally suppressed (Figure 1) (6). 
HIV Cascade of care in the EMR

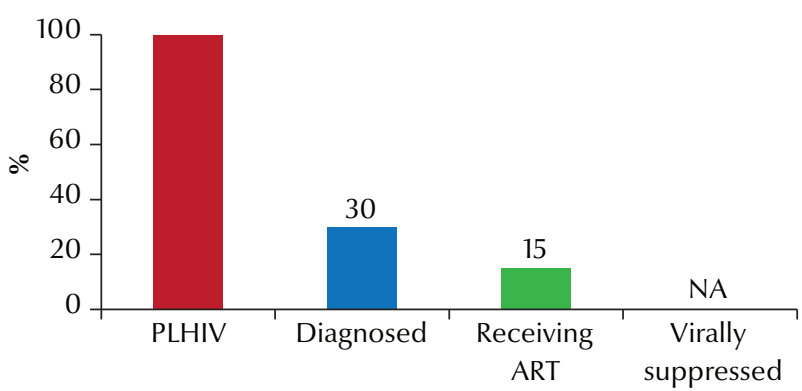

Figure 1 HIV diagnosis, care and viral suppression cascade in the EMR as of end 2017.

The missed opportunities to diagnose more PLHIV, diagnosing them early and properly linking of them to care and treatment are compromising the gains from investments made in
HIV response to date in the Region. In spite of increased efforts to scale up HIV testing, treatment and care services, the EMR is still facing significant challenges in increasing their coverage of services.
Overcoming those challenges requires, among other things, openness to nontraditional approaches to HIV testing service delivery; bringing the services to the community level with involvement of the civil society; and the use of new technologies such as self-testing and diversify HIV testing service delivery approaches to meet the needs of those left behind. Furthermore, in order to optimize the benefit of testing, the Region needs to invest in ensuring early diagnosis as well as efficient linkages of those diagnosed HIV positive to care and treatment.

Rapidly scaling up HIV testing services remains a paramount necessity for the EMR; not just to meet the global targets, but also to ensure the fundamental human right to health for PLHIV.

\section{References}

1. Vella S, Schwartländer B, Sow S, Eholie, RL Murphy. The history of antiretroviral therapy and of its implementation in resourcelimited areas of the world. AIDS. 2012;26(10):1231-1241. doi: 1097/QAD.0b013e32835521a3.

2. UNAIDS. UNAIDS announces that the goal of 15 million people on life-saving HIV treatment by 2015 has been met nine months ahead of schedule. Geneva: UNAIDS; 14 July 2015 (http:// www.unaids.org/en/resources/presscentre/pressreleaseandstatementarchive/2015/july/20150714_PR_MDG6report, accessed 29 October 2017).

3. UNAIDS. UNAIDS data 2017. Geneva: UNAIDS; 2017 (http:// www.unaids.org/sites/default/files/media_asset/20170720_ Data_book_2017_en.pdf, accessed 29 October 2017).
4. UNAIDS. Global AIDS Update 2016. Geneva: UNAIDS; 2016 (http://www.unaids.org/sites/default/files/media_asset/ global-AIDS-update-2016_en.pdf, accessed 29 October 2017).

5. WHO Regional Office for the Eastern Mediterranean (EMRO). Test-treat-retain cascade assessment reports from Sudan, Punjab-Pakistan, Morocco, Egypt, Lebanon and Islamic Republic of Iran. Cairo: EMRO [Unpublished].

6. WHO Regional Office for the Eastern Mediterranean (EMRO). Regional surveillance monitoring report 2017. Cairo: EMRO [unpublished]. 